\title{
Editorial
}

\section{Tracking Effects of Exercise on Neuronal Plasticity}

\author{
Henriette van Praag ${ }^{\mathrm{a}}$ and Brian Christie ${ }^{\mathrm{b}}$ \\ ${ }^{a}$ National Institute on Aging, Baltimore, USA \\ ${ }^{\mathrm{b}}$ University of British Columbia, Vancouver, Canada
}

The inaugural issue of Brain Plasticity includes ten papers that focus on the effects of exercise on brain function in animal models. Exercise is a simple, lowcost intervention that promotes cognition and mood, protects against damage associated with neurodegeneration and may alleviate drug addiction in humans. The mechanisms underlying the beneficial effects of physical activity are the subject of intense investigation. For this Special Issue we have invited scientists working on basic research into the cellular, molecular and behavioral changes associated with running. We are grateful for their excellent and insightful contributions.

Several papers in this issue focus on the cellular mechanisms underlying beneficial effects of running on cognition and mood. The article by Sama Sleiman and Moses Chao describes the historical background and discovery of neurotrophic factors, their role (especially of BDNF) in synaptic plasticity and potential clinical implications of their modulation by exercise. The article also addresses how peripheral factors, such as ketones and myokines, may be implicated in the running-induced upregulation of BDNF [8]. The role of a specific myokine, irisin, a cleavage product derived from a muscle protein FNDC5 that is secreted into circulation, and its role in upregulation of brain BDNF levels by exercise is discussed in detail by Christiane Wrann [10]. In a comprehensive review, Simone Bolijn and Paul Lucassen describe how peripheral factors such as insulin-like growth factor, adiponectin and AMP-kinasethat may regulate exercise-induced hippocampal cell genesis [1]. Indeed, a focus on the effects of exercise on adult neurogenesis is the subject of several papers in this issue. Stefano FarioliVecchioli and Felice Tirone review the cell-cycle kinetics in both neurogenic niches the dentate gyrus and subventricular zone, and discuss how runningdependent enhancement of proliferation may depend on changes in cell cycle length in select types of neural progenitor cells [3].

Two research reports in this issue address effects of running on behavior. Justin Rhodes and his colleagues test a hybrid transgenic mouse model with reduced neurogenesis on hippocampus-dependent and non-dependent tasks and the response of this strain to exercise [4]. Furthermore, Joseph Bischofberger and co-workers provide novel evidence in their research report for running-induced enhanced pattern separation using a new behavioral approach with highly similar novel and familiar objects that does not cause stress or requires food or water deprivation of the animals, thereby highlighting the effects of exercise on cognition. They also show that running increases the dendritic outgrowth into the outer molecular layer and the number of young, double cortin-positive granule cells in the dorsal rather than in the ventral dentate gyrus [2].

The subject of another set of papers in this Special Issue is the effects of exercise on neurodegenerative disease, stress and drug addiction. Brian Christie and his colleagues [6] give a historical perspective on hippocampal structure, function and plasticity, followed by an overview of effects of exercise on the hippocampus under normal and disease conditions including Fetal Alcohol Syndrome, Traumatic brain injury, Stroke and neurodegenerative diseases (Alzheimer's, Parkinson's and Huntington's Disease). Giselle Petzinger and her colleagues contribute a focused review on the impact of exercise on neuroplasticity in animal models of Parkinson's disease. 
Modulation of dopaminergic and glutamatergic transmission, synaptogenesis and cerebral blood flow in the basal ganglia, prefrontal cortex and cerebellumby exercise are discussed [7]. Hideaki Soya and his colleagues focus on the dosing of exercise and provide evidence that mild exercise is the most beneficial for brain function [5]. Another aspect of the effects of exercise is highlighted by Chitra Mandyam and her colleagues in a comprehensive review on methamphetamine addiction and associated neurotoxicity.

\section{REFERENCES}

[1] Bolijn S, Lucassen PJ. How the body talks to the brain; peripheral mediators of physical activity-induced proliferation in the adult hippocampus. Brain Plasticity. 2015;1:1-23.

[2] Bolz L, Heigele S, Bischofberger J. Running improves pattern separation during novel object recognition. Brain Plasticity. 2015;1:125-37.

[3] Farioli-Vecchioli S, Tirone F. Control of the cell cycle in adult neurogenesis and its telation with physical exercise. Brain Plasticity. 2015;1:37-50.

[4] Hamilton GF, Majdak P, Miller DS, Bucko PJ, Merritt JR, Krebs CP, Rhodes JS. Evaluation of a C57BL/6J x 129S1/ SvImJ hybrid nestin-thymidine kinase transgenic mouse model for studying the functional significance of exerciseinduced adult hippocampal neurogenesis. Brain Plasticity. 2015;1:79-91.

[5] Okamoto M, Yamamura Y, Liu Y-F, Min-Chul L, Matsui T, Shima T, Soya M, Takahashi K, Soya S, McEwen BS, Soya H. Hormetic effects by exercise on hippocampal neurogenesis with glucocorticoid signaling. Brain Plasticity. 2015;1: 145-54.

[6] Patten AR, Yau SY, Fontaine CJ, Meconi A, Wortman RC, Christie BR. The benefits of exercise on structural and functional plasticity in the rodent hippocampus of different disease models. Brain Plasticity. 2015;1:93-123.

[7] Petzinger GM, Holschneider DP, Fisher BE, McEwen S, Kintz N, Halliday M, Toy W, Walsh JW, Beeler J, Jakowec MW. The effects of exercise on dopamine neurotransmission in Parkinson's disease: targeting neuroplasticity to modulate basal ganglia circuitry. Brain Plasticity. 2015;1:25-35.

[8] Sleiman SF, Chao MV. Downstream consequences of exercise through the action of BDNF. Brain Plasticity. 2015; $1: 139-44$.

[9] Somkuwar SS, Staples MC, Fannon MJ, Ghofranian A, Mandyam CD. Evaluating exercise as a therapeutic intervention for methamphetamine addiction-like behavior. Brain Plasticity. 2015;1:59-77.

[10] Wrann CD. FNDC5/Irisin - Their role in the nervous system and as a mediator for beneficial effects of exercise on the brain. Brain Plasticity. 2015;1:51-7. 